\title{
Sustained change blindness to incremental scene rotation: A dissociation between explicit change detection and visual memory
}

\author{
ANDREW HOLLINGWORTH \\ University of Iowa, Iowa City, Iowa \\ and \\ JOHN M. HENDERSON \\ Michigan State University, East Lansing, Michigan
}

\begin{abstract}
In a change detection paradigm, the global orientation of a natural scene was incrementally changed in $1^{\circ}$ intervals. In Experiments 1 and 2, participants demonstrated sustained change blindness to incremental rotation, often coming to consider a significantly different scene viewpoint as an unchanged continuation of the original view. Experiment 3 showed that participants who failed to detect the incremental rotation nevertheless reliably detected a single-step rotation back to the initial view. Together, these results demonstrate an important dissociation between explicit change detection and visual memory. Following a change, visual memory is updated to reflect the changed state of the environment, even if the change was not detected.
\end{abstract}

The visual world is constantly changing around us, often in salient ways, such as the flow of traffic and pedestrians on a busy street, but also in more subtle ways, such as the gradual change in illumination produced by a cloud passing in front of the sun. In addition to changes in the world, changes in visual input are constantly generated by observer movements, such as shifts of the eyes and head. To what extent does the visual system keep track of the current state of the visual environment, and what role does explicit awareness of change play in updating visual representations to reflect changed conditions? Consider the following real-world example. One meets a person in the morning, and later that day, one meets that person again. In the interval, he has had his hair cut, but one does not explicitly notice that his hair has changed. How, then, is this person represented in visual memory? Perhaps explicit awareness of change is necessary to update visual memory. If one does not notice that the hair had changed, the original memory is retained, and the more recent information is discarded. Alternatively, memory may come to reflect the changed hair in the absence of any explicit awareness that the person has changed. Other possibilities arise,

This research was supported by NIH Grant R03 MH65456 to A.H. and NSF Grants SBR 9617274 and ECS 9873531 to J.M.H. Aspects of this study were reported at the 2nd Annual Meeting of the Vision Sciences Society, Sarasota, FL, 2002. We thank Diego Fernández-Duque, Steve Mitroff, Brian Scholl, and Dan Simons for helpful discussions of the present research. Correspondence concerning this article should be addressed to A. Hollingworth, Department of Psychology, University of Iowa, 11 Seashore Hall E, Iowa City, IA 52242-1407 (e-mail: andrewhollingworth@uiowa.edu). such as a combination or averaging of the two representations. Since we live in dynamically changing environments, understanding how representations are updated with changing conditions is central to understanding realworld vision and memory.

In the present study, we examined the updating of visual memory representations when a change is introduced gradually in a real-world scene. Gradual incremental changes provide a potentially fruitful method for investigating visual updating. First, incremental change is characteristic of many real-world circumstances, such as the example cited above of a cloud passing in front of the sun. Second, incremental changes may be particularly difficult to notice explicitly (Simons, Franconeri, \& Reimer, 2000), allowing examination of potential updating in the absence of explicit awareness that a change has occurred. In three experiments, we examined participants' sensitivity to gradual changes in the global orientation of natural scenes and asked whether, in the absence of explicit awareness of change, visual memory is implicitly updated to reflect the changed orientation of the scene.

Initial evidence suggests that complex scenes are represented in a viewpoint-dependent manner (Chua \& Chun, 2003; Diwadkar \& McNamara, 1997; Simons \& Wang, 1998), yet little is known about how precisely global orientation is represented. To examine visual updating across gradual changes in global orientation, we rotated images of complex scenes progressively in $1^{\circ}$ intervals. Experiment 1 tested participants' sensitivity to the incremental in-depth rotation of an entire living room scene. Experiment 2 replicated Experiment 1, but with a picture plane rotation of an office scene that eliminated 


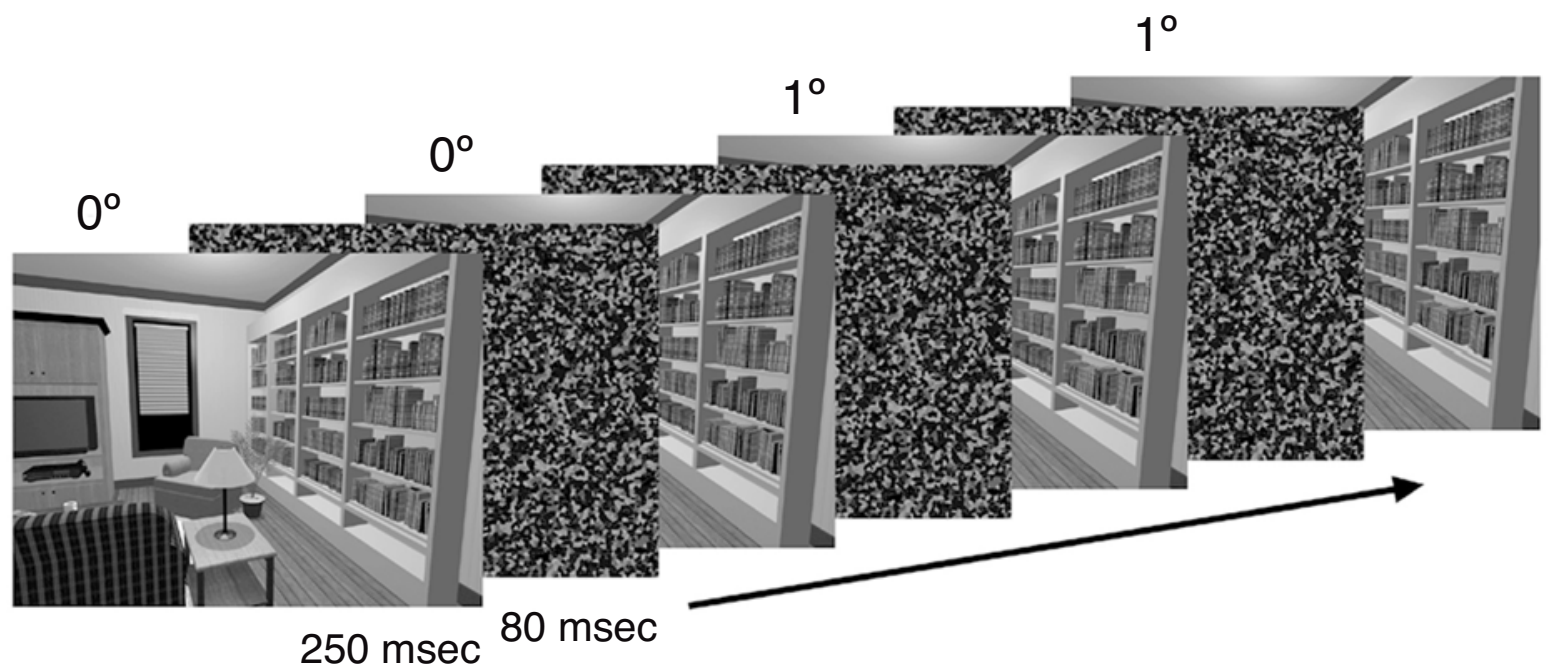

Figure 1. Sequence of events in a trial in Experiment 1. Each scene viewpoint was displayed twice for $250 \mathrm{msec}$, with each image separated by an 80-msec pattern mask. The sequence of views was continued until the participant responded or until all viewpoints had been displayed.

the appearance and disappearance of local objects during rotation. In both experiments, participants demonstrated sustained change blindness to incremental rotation, often coming to consider a significantly different scene viewpoint as an unchanged continuation of the original view. Experiment 3 tested whether memory is updated with incremental rotation to reflect a recent view (or views) in the absence of explicit change detection. The office scene was incrementally rotated, followed by a single-step rotation back to the initial image. Participants who did not detect the incremental rotation nonetheless reliably detected the single-step rotation back to the initial image, demonstrating that memory had been implicitly updated with incremental rotation to reflect the changed state of the environment.

\section{EXPERIMENT 1}

To investigate sensitivity to incremental scene rotations, we used a version of the flicker paradigm developed by Rensink, O'Regan, and Clark (1997; see Figure 1). The stimuli were a series of viewpoints rendered from a threedimensional (3-D) scene model. Consecutive viewpoints differed by $1^{\circ}$ of orientation in depth. Each viewpoint was displayed twice for $250 \mathrm{msec}$, and each image was separated by an 80 -msec pattern mask. The series of images was similar to what one would perceive if one slowly turned within a scene to face a different direction. The participants were instructed to press a button if they noticed the scene change in any way whatsoever. After detection, the participants described the change. Note that in this method, the entire scene changes, so change detection failure could not arise from failure to attend a changing region, as was the case with recent work by Simons et al. (2000), who introduced gradual changes to local scene regions. Due to the possibility that successful change detection would influence performance on subsequent trials, each participant completed one trial.

\section{Method}

Participants. Thirty-two participants from the Michigan State University community completed the experiment. All the participants reported 20/20 uncorrected or corrected vision.

Apparatus. The stimuli were displayed on a Sony Trinitron video monitor operating at $100 \mathrm{~Hz}$. Responses were collected on a serial button box. A 486-based PC-compatible computer controlled stimulus presentation and recorded button responses.

Stimuli. For the in-depth rotation in Experiment 1, a living room scene was rendered from a "camera" position in the center of the 3-D model. The camera was then rotated in depth in $1^{\circ}$ intervals, with a new image rendered at each viewpoint. Eighty-two images were created, spanning $81^{\circ}$ of rotation. The pattern mask appearing between scene stimuli was made up of a dense assortment of random contours. Images were displayed at a resolution of $800 \times 600$ pixels by 256 colors and subtended $23^{\circ}$ (width) $\times 16^{\circ}$ (height) at a viewing distance of $64 \mathrm{~cm}$. Viewing distance was maintained by a forehead rest.

Procedure. The scene stimuli were presented in sequence, starting at $0^{\circ}$. Each viewpoint was presented twice, for $250 \mathrm{msec}$ each, with each image separated by an $80-\mathrm{msec}$ pattern mask. Thus, orientation was changed $1^{\circ}$ every $660 \mathrm{msec}$. The participants were told that an image of a real-world scene would flash on and off. They were instructed to press a response button as soon as they noticed the scene change "in any way whatsoever." They were told that the flashing of the scene, on and off, should not be considered a change. These instructions were quite general and did not inform the participants of the nature of the possible change. If the participants had known that the scene might rotate, they potentially could have used undesirable strategies to detect it (such as fixating an object and waiting to see whether it remained at fixation or monitoring the edge of the screen for the accretion or decretion of objects). A button press terminated the trial, and the participant wrote a brief description of the change. If the participant did not respond, the trial terminated after all viewpoints had been displayed. Elapsed rotation before detection was recorded. 

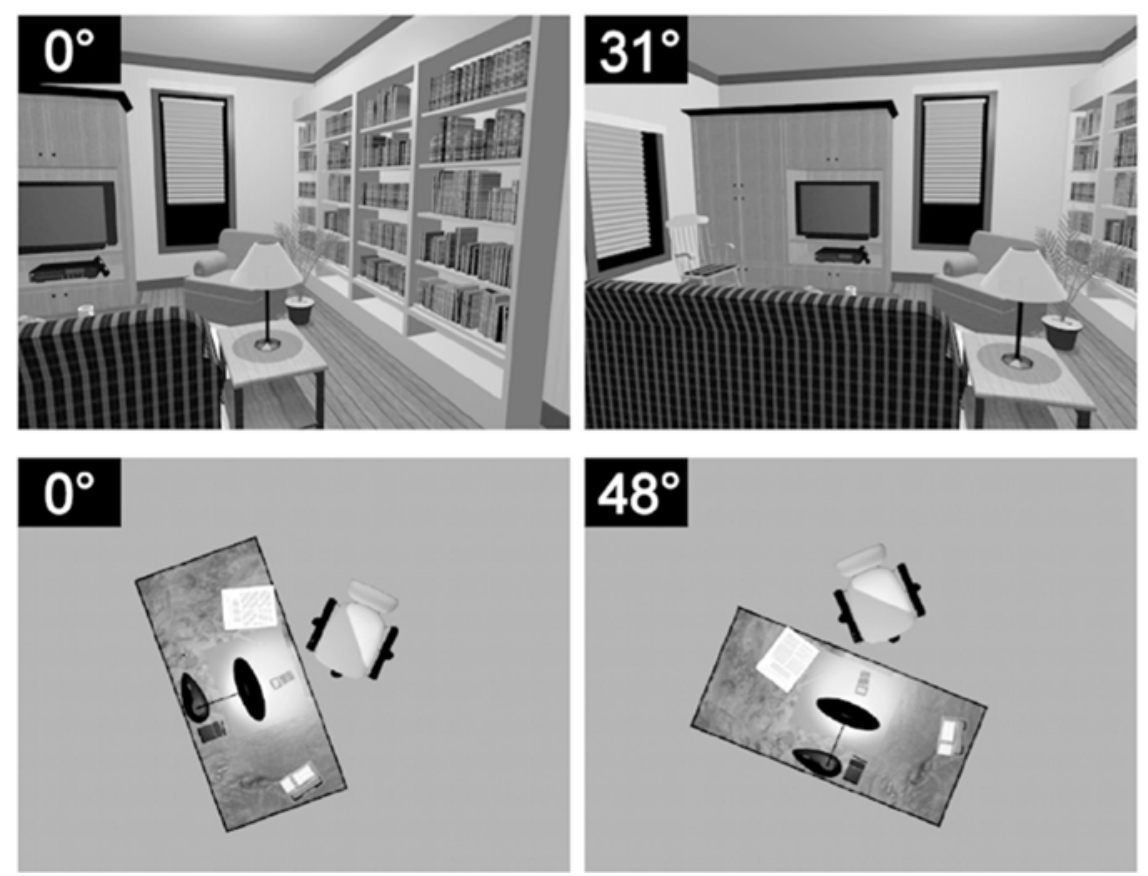

Figure 2. Initial $0^{\circ}$ viewpoint and median viewpoint at change detection in Experiment 1 (upper panels) and Experiment 2 (lower panels).

\section{Results and Discussion}

Four of the 32 participants were eliminated because they either failed to understand the instructions or falsely described the change. Descriptions needed to be clearly false to be eliminated (e.g., "couch turned to stripes").
For the remaining 28 participants, median rotation before detection was $31^{\circ}$. Figure 2 displays the initial image $\left(0^{\circ}\right)$ and the $31^{\circ}$ image. At $660 \mathrm{msec} /{ }^{\circ}, 31^{\circ}$ of rotation took approximately $20 \mathrm{sec}$. Figure 3 shows the cumulative percentage of participants detecting a change as a function of

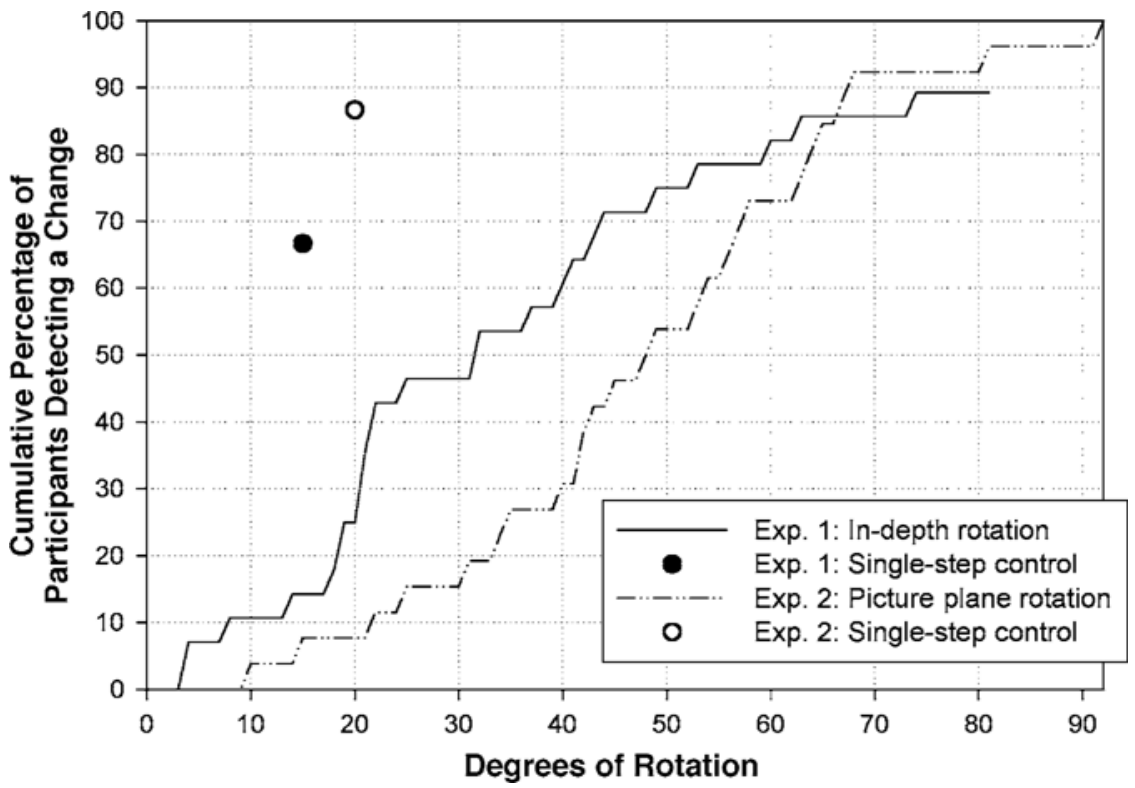

Figure 3. Change detection performance for Experiments 1 and 2. Lines represent the cumulative percentages of participants detecting a change as a function of the elapsed rotation. The single data points plot the percentages of participants detecting a change in the singlestep control experiments. 
the elapsed rotation. Not only did half the participants fail to detect the change for $31^{\circ}$ of rotation, 3 participants failed to detect the change at all, even though the rotation continued to $81^{\circ}$ and most of the original objects had rotated out of view. These results demonstrate that a series of small changes in the in-depth orientation of a scene can be additive to the point that a significantly different view of the scene is considered an unchanged continuation of the initial view. This sustained change blindness to incremental rotation was observed even though change detection was the participant's only task and ample time was available for detection.

Although the participants exhibited sustained insensitivity to incremental rotation, all but 3 eventually did detect the change. The manner in which changes were eventually detected provides evidence about the types of information the participants were comparing across views. The data do not allow us to specify the precise information supporting ultimate change detection, since the entire scene was changing and there were many possible routes to detection. There appear to be three broad possibilities, however. First, the participants may have attended a local object in the scene and monitored for a change in that object, shifting attention to a different object when no change was detected (an object-by-object comparison hypothesis). Second, the participants may have compared information about the global layout of objects in the scene (a global comparison hypothesis). Finally, the participants may have detected the change by noticing a difference in the inventory of objects present in the scene, such as the appearance or disappearance of an object (an inventory change hypothesis).

The change descriptions provided by the participants after detection can help identify which of these hypotheses best accounts for ultimate change detection. Of the 25 participants detecting a change, 9 reported the global change alone (e.g., "the room was rotating right"), 3 reported global change along with a change in object inventory (e.g., "the picture seemed to travel across the room; a chair appeared"), 2 reported global change along with a change in the properties of an individual object (e.g., "the view of the room was moving toward the left; less of the bookcase could be seen as the flickering occurred"), 4 reported a change to multiple objects (e.g., "coffee cup and other objects were sliding or moving to the right of the screen"), 5 reported a change in object inventory only (e.g., "a chair appeared on the left-hand side of the screen"), and 2 reported a change only in the properties of a local object (e.g., "the chair on the left began to rotate"). In addition, there was a spike in detection performance between approximately $17^{\circ}$ and $24^{\circ}$, when the first new object, a rocking chair, appeared. Of the 9 participants detecting a change between $17^{\circ}$ and $24^{\circ}$ of rotation, 7 mentioned the chair in their description, and the chair was not reported in any other participants' descriptions. Thus, the appearance of a new object appears to be relatively salient evidence for change.
To summarize the change description data, 18 of the 25 participants who detected a change reported global change or change to multiple objects, consistent with a global comparison hypothesis. Eight participants reported a change in object inventory. Four participants reported a change in the properties of an individual object. ${ }^{1}$ Although the participants may possibly have used an objectby-object comparison strategy, the ultimate detections seem to have been generated primarily by detection of changes in the global organization of the scene and changes in object inventory. The preponderance of detections based on global, rather than object-by-object, comparison is consistent with prior evidence that changes in global layout are detected more reliably than changes in individual object properties (Simons, 1996).

In the remainder of this article, we will argue that despite failures of explicit change detection for incremental rotation, the visual memory representation of the scene was nevertheless sensitive to the difference between views. Specifically, consecutive views of the scene were compared, and although the difference between views was not sufficient to yield explicit change detection, it was sufficient to update memory to reflect the changed viewpoint. $^{2}$ Before directly examining whether memory was updated to reflect recent views (Experiment 3), it is important to eliminate an alternative explanation for the poor explicit change detection in Experiment 1. Changes may have been missed because the participants simply failed to construct or retain a representation from a previous view (O'Regan, 1992; O'Regan \& Noë, 2001). To demonstrate that scene information is indeed retained and compared across consecutive views, we ran a control experiment. In the control experiment, a single-step rotation was introduced that was likely to be detectable but that was much smaller than the median rotation necessary to detect the incremental change. The living room scene was rotated in depth in one step from $0^{\circ}$ to $15^{\circ}$. To avoid introducing the change right at the beginning of the trial, the initial view was displayed five times before presentation of the changed view, which was then displayed six times to allow time for response. Otherwise, the method was identical to that in Experiment 1.

One of the 15 participants in the control experiment incorrectly described the change. Of the remaining 14 participants, 9 detected and correctly described the single-step rotation of $15^{\circ}$ (64\%; see Figure 2$)$. In Experiment 1, only $14 \%$ of the participants had detected the incremental change by $15^{\circ}$ of rotation. Six of the 9 participants who detected the change reported the global change, 1 a change to multiple objects, and 2 a change to the properties of a single object. These data demonstrate that a representation of a previous scene viewpoint is indeed retained across the masked interval, that it is then compared with perceptual information from the current view, and that the results of this comparison can be explicitly available. Without any one of these component processes, successful detection of the single-step rotation could not have occurred. 


\section{EXPERIMENT 2}

Experiment 2 replicated Experiment 1 with a stimulus set in which the same objects were always visible, eliminating the opportunity to detect global changes by noting changes in object inventory, particularly the appearance of a new object. An office scene stimulus was used, which was rotated incrementally counterclockwise in the picture plane. In addition to eliminating the appearance and disappearance of objects, the picture plane rotation corrected a possible weakness in the Experiment 1 method. In-depth rotation, as in Experiment 1, could possibly be interpreted as a consequence of a change in viewer orientation, rather than a change to the scene itself. The participants may have been hesitant to report in-depth rotation as a change to the scene because they interpreted the rotation as just a different observer viewpoint within a static environment. In Experiment 2, however, picture plane rotation could not plausibly be interpreted as a change in observer viewpoint.

\section{Method}

Participants. Thirty-nine participants from the Yale University community completed the experiment. All the participants reported 20/20 uncorrected or corrected vision.

Apparatus. The stimuli were displayed on a Sony Trinitron video monitor operating at $100 \mathrm{~Hz}$. Responses were collected on a serial button box. A Pentium 3 based PC-compatible computer controlled stimulus presentation and recorded button responses.

Stimuli. An office scene consisting of a desk, a chair, and assorted objects was rendered from a top-down view. A total of 360 separate views were created in a counterclockwise sequence, spanning all $360^{\circ}$ in the picture plane. The pattern mask appearing between scene stimuli was made up of a dense assortment of colored shapes. Images were displayed at a resolution of $800 \times 600$ pixels by 24 -bit color and subtended $23^{\circ}$ (width) $\times 17^{\circ}$ (height) at a viewing distance of $80 \mathrm{~cm}$. Viewing distance was maintained by a forehead rest.

Procedure. The procedure was the same as that in Experiment 1.

\section{Results and Discussion}

Of the 39 participants, 13 were eliminated ( 5 failed to understand the instructions; 8 falsely described the change). For the remaining 26 participants, median rotation before detection was $48^{\circ}$ (see Figures 2 and 3 ), which took approximately $32 \mathrm{sec}$. After lengthy viewing without detection, some participants apparently felt compelled to report a change, producing the relatively large number of false reports. Of the 26 detections, 15 participants described the entire scene rotation (e.g., "the entire image began shifting at an angle, as if on a round table that was spinning very slowly"), and 4 reported a change in the properties of a single object (e.g., "the chair rotated anticlockwise"). The remaining 7 participants reported a change to the desk (e.g., "desk is moving"). Reports of desk rotation are difficult to assess on the global/local dimension, because the desk was the primary element in the scene and a number of objects were located on its surface. A desk rotation report could plausibly refer to a rotation of the entire "desk scene," the "desk and the ob- jects on it," or "the desk alone." The last interpretation is the least plausible, since a rotation of the desk alone would have left some of the objects without support.

Overall, the results of Experiment 2 replicate those in Experiment 1. The participants demonstrated sustained insensitivity to the global rotation of the scene, even though change detection was the only task and the participants viewed the changing stimulus for over $30 \mathrm{sec}$, on average, prior to detection. When the participants did finally detect the change, most reported global rotation, consistent with the global comparison hypothesis.

As in Experiment 1, we completed a control experiment to ensure that change blindness in Experiment 2 was not observed because the participants did not form a memory representation of the scene (O'Regan, 1992; O'Regan \& Noë, 2001) or because that memory representation was not compared with perceptual information from a subsequent image. For the Experiment 2 control, the office scene was rotated in one step from $0^{\circ}$ to $20^{\circ}$. To avoid introducing the change right at the beginning of the trial, the initial view was displayed five times before presentation of the changed view, which was then displayed six times to allow time for response. Otherwise, the method was identical to that in Experiment 2. Thirteen of the 15 participants in the control experiment detected and correctly described the single-step rotation of $20^{\circ}(87 \%)$. In Experiment 2, only $8 \%$ of the participants had detected the incremental change by $20^{\circ}$ of rotation. Three participants reported whole-image rotation, 8 reported rotation of the desk, and 2 reported a change in a single local object.

The control data in Experiments 1 and 2 demonstrate that insensitivity to incremental rotation in the main experiments was observed despite the fact that consecutive views are indeed compared and despite scene memory representations of sufficient fidelity to detect much smaller changes than those required under incremental conditions. Sustained change blindness to incremental rotation is, therefore, unlikely to have been caused by a lack of visual memory. In Experiment 3, we investigated the counterintuitive possibility that the effect arises because visual memory is instead quite sensitive to difference between views, updating memory to reflect the changed state of the environment, in the absence of explicit change detection.

\section{EXPERIMENT 3}

In Experiment 3, we directly tested whether, in the absence of explicit change detection, visual memory is updated to reflect the changed state of the environment. One logical possibility is that memory is not updated unless one has explicit evidence that the scene has changed. Under this explicit updating hypothesis, during a trial in Experiments 1 and 2, the $1^{\circ}$ image was compared with memory for $0^{\circ}$. If the change was not explicitly detected, memory for the scene was not altered. Next, the $2^{\circ}$ image was compared with memory for $0^{\circ}, 3^{\circ}$ with memory for $0^{\circ}$, and so on. With multiple rotations, the current perceptual information and the visual memory representation 
would become increasingly dissimilar. The second possibility is that visual memory for the scene is incrementally updated with changes in the environment, even in the absence of explicit change detection. Under this implicit updating hypothesis, the $1^{\circ}$ image was compared with memory for $0^{\circ}$. No change was explicitly detected, but memory was implicitly updated to reflect information in the $1^{\circ}$ image. Across multiple small changes in viewpoint, memory would be incrementally updated to reflect a recent view (or views). As a result, the discrepancy between current perceptual information and scene memory would always remain very small.

Experiments 1 and 2 provide initial evidence in support of the implicit updating hypothesis. The sustained change blindness to incremental rotation suggests that comparison processes were generally operating over similar representations, consistent with the possibility that visual memory was implicitly updated to reflect recent views.

To test these hypotheses directly, in Experiment 3 the office scene was incrementally rotated from $0^{\circ}$ to either $20^{\circ}$ or $30^{\circ}$. At this point, the scene rotated back to $0^{\circ}$ in a single step. The two updating hypotheses make markedly different predictions in this paradigm. If visual memory is updated only when a change is explicitly detected, participants who have not explicitly detected the incremental change should not detect the shift back to $0^{\circ}$, because perceptual information from the final image $\left(0^{\circ}\right)$ would be in precise correspondence with visual memory (also for $0^{\circ}$ ). However, if visual memory is incrementally updated in the absence of explicit change detection, when the scene is rotated back to $0^{\circ}$, the change should be salient, because it would be memory for the most recent views compared with perceptual information from $0^{\circ}$. That is, participants who do not explicitly detect the incremental change should nevertheless notice when the scene abruptly returns to its original orientation.

\section{Method}

Participants. Sixty-one participants from the Yale University community completed the experiment. All the participants reported 20/20 uncorrected or corrected vision.

Procedure. The office scene stimuli were presented in $1^{\circ}$ intervals from $0^{\circ}$ to either $20^{\circ}$ or $30^{\circ}$. At this point, the $0^{\circ}$ image was again displayed and was repeated five times to ensure that the participants had an opportunity to respond before the end of the trial. Otherwise, the procedure was identical to that in Experiment 2.

\section{Results and Discussion}

$\mathbf{2 0}^{\circ}$ condition. Of the 30 participants, 5 were eliminated because they pressed the button during the incremental rotation (1 correctly described the change). Four additional participants were eliminated because they did not respond initially but reported noticing a change during debriefing. Of the 21 remaining participants, 14 detected the rotation back to $0^{\circ}(66.7 \%)$. Of these 14,5 reported the whole-image change, 1 a change to multiple objects, 4 a change to the desk, and 4 a change to a single local object. Nine participants reported the direction of rotation. Eight of these reported clockwise rotation (consis- tent with the single-step rotation back to $0^{\circ}$ ); 1 reported counterclockwise rotation.

$30^{\circ}$ condition. Of the 31 participants, 10 were eliminated because they pressed the button during the incremental rotation (5 correctly described the change). One additional participant was eliminated because he did not press a button but reported noticing a change during debriefing. Of the 20 remaining participants, all 20 detected the rotation back to $0^{\circ}$. Nine participants reported the whole-image change, 8 a change to the desk, and 3 a change to a single local object. Fifteen participants reported the direction of rotation. Fourteen of these reported clockwise rotation (consistent with the single-step rotation back to $0^{\circ}$ ); 1 reported counterclockwise rotation.

In Experiment 3, the participants who did not explicitly detect the incremental rotation reliably detected the single-step rotation back to the initial image, with the large majority reporting a direction of rotation consistent with that single-step change. The participants described seeing a single change at the end of the trial, unaware that the scene had rotated incrementally and that the final image was, in fact, the initial image. The only way that the rotation back to the initial image could have been detected is if visual memory was updated with incremental rotation to reflect a more recent view (or views). If memory had not been updated or was absent, the final rotation would not have been detectable. Thus, Experiment 3 provides strong support for the implicit updating hypothesis.

On the face of it, successful detection of the rotation back to the initial view may seem unsurprising, since the single-step rotation was quite large. And indeed, such a rotation would be obvious if it occurred as an immediate transition (without a masked interstimulus interval [ISI]). But with a masked ISI between images, detection must have depended on visual memory, and furthermore, detection must have depended on the updating of memory to reflect a recent view (or views). Consider the following real-world example. Imagine that the visible world dims very gradually as the wispy leading edge of a cloud passes in front of the sun. Quite possibly, the gradual dimming would go undetected. Shortly afterward, the visible world brightens quickly and significantly as the sun abruptly reemerges. It would not be at all surprising if people explicitly detected the abrupt brightening. But if the brightening occurred during a perceptual disruption analogous to the masked ISI, such as a blink, it seems much less obvious that the abrupt change back to the original illumination would be detected, since detection would depend on visual memory being implicitly updated to reflect the dimmed state of the environment.

\section{GENERAL DISCUSSION}

In the present study, we investigated participants' sensitivity to a series of small changes in the global orientation of a scene. In Experiments 1 and 2, the participants demonstrated sustained change blindness to incremental 
scene rotation, coming to perceive significantly different views of a scene as an unchanged continuation of the initial view. This effect would not be particularly surprising if the participants simply failed to remember previous views (e.g., O'Regan, 1992; O'Regan \& Noë, 2001) or failed to compare information from one view with that from the next. However, control experiments showed successful detection of much smaller total rotations when the change was introduced in a single step, demonstrating that information from a previous view was retained and was reliably compared with perceptual information from the current view. In addition, the majority of the participants reported global rotation, suggesting that these comparisons typically included information about global orientation. Thus, in the incremental conditions, we can be confident that consecutive views were indeed remembered and compared. Although the product of these comparisons was rarely sufficient to support explicit change detection (the participants were not aware that different consecutive views were not equivalent), different views were not treated as equivalent in visual memory. Experiment 3 provided direct evidence that visual memory was implicitly updated to reflect recent scene information. If consecutive views had been equivalent in visual memory, there would have been no informational basis upon which to update memory to reflect more recent views. This dissociation between the updating of visual memory and explicit change detection helps explain the sustained change blindness effect itself. Because memory was incrementally updated to reflect recent views, each comparison between scene memory and current perceptual information operated over similar representations, even though both of these representations were often significantly different from the initial image. ${ }^{3}$

The present data contribute to a growing body of evidence demonstrating that explicit change detection underestimates the sensitivity of visual memory (FernándezDuque \& Thornton, 2000; Hayhoe, Bensinger, \& Ballard, 1998; Henderson \& Hollingworth, 2003a; Hollingworth \& Henderson, 2002; Hollingworth, Williams, \& Henderson, 2001; Williams \& Simons, 2000). ${ }^{4}$ For example, Hollingworth et al. (2001) found that when participants failed to explicitly report a change in a natural scene, fixation durations on changed objects were significantly elevated, as compared with a no-change control. Such results falsify the hypothesis that explicit change detection provides an exhaustive measure of visual memory (Rensink et al., 1997). Demonstrations of change blindness therefore cannot be taken as strong evidence that visual scene memory is absent (see also Simons, 2000). In fact, the reverse appears to be true. Despite evidence of change blindness, visual memory representations of natural scenes preserve a great deal of visual information. Scene representations are certainly not as veridical as a photograph (Henderson \& Hollingworth, 2003b; Irwin, 1991) but are of sufficient fidelity to reliably code the visual properties of large numbers of constituent objects
(Hollingworth, 2003, 2004; Hollingworth \& Henderson, 2002). The present data extend these findings to demonstrate that visual memory is also sensitive to relatively small differences in the global orientation of a scene.

In addition, the present results suggest that a threshold mechanism may govern the explicit detection of changes in the global orientation of a scene. Visual memory was sensitive to the difference between views, but the participants rarely detected the $1^{\circ}$ changes explicitly. Apparently, the visual system tolerates small differences in orientation, without triggering explicit awareness of change. Only when much larger rotations occurred in a single step did the participants reliably detect the change. During normal visual interaction with the world, small discrepancies between the expected orientation and the viewed orientation of a scene may arise due to inaccuracies in sensorimotor processes. If an eye movement under- or overshoots the target of the eye movement, the resulting scene image will have an orientation slightly different from that produced after an accurate eye movement. In addition, small orientation discrepancies across adjacent views would be produced if head movements were not precisely accounted for during an eye movement or if either head or eye movements were not precisely accounted for during a blink. Since it is exceedingly unlikely that a natural scene would itself rotate from one view to the next, the visual system may be biased toward attributing small discrepancies in viewpoint to internal error, rather than to external change, producing the present failures of explicit change detection.

A threshold mechanism minimizes the possibility that small internal errors will be falsely attributed to a change in the world. However, in the rare case that a small discrepancy is actually the result of external change, it will be falsely attributed to internal error, yielding change blindness. Therefore, a threshold mechanism for explicit change detection helps explain how change blindness can occur despite accurate visual memory. The visual system is sensitive to the difference between views, but participants do not explicitly detect the change unless the difference exceeds threshold (see Hollingworth, 2003, and Hollingworth \& Henderson, 2002, for further discussions of how change blindness arises despite accurate visual memory). It is likely that the amount of evidence for change varied from comparison to comparison during gradual rotation, depending on whether the participant was attending information in the scene that was diagnostic of rotation. Although evidence of change was typically not sufficient to exceed threshold for explicit detection, evidence of change must have been sufficient to exceed threshold on some rare comparisons, because almost all the participants did eventually detect the change.

Finally, how is visual memory updated with incremental rotation to reflect recent views? There appear to be three main possibilities. First, a single scene representation may be modified with incremental rotation to reflect the most recent scene view. Under this replace- 
ment hypothesis, a representation of the previous view is retained and compared with perceptual information from the current view; after comparison, however, memory for the previous view is replaced by memory for the current view. Although possible, we do not consider this replacement hypothesis particularly plausible, given evidence of preserved memory for the original version of a stimulus after an undetected change (Hollingworth \& Henderson, 2002; Mitroff, Simons, \& Levin, in press; Simons, Chabris, Schnur, \& Levin, 2002; see also McCloskey \& Zaragoza, 1985). A second possibility is that multiple views are stored in memory, consistent with instance theories of viewpoint-dependent object and scene recognition (e.g., Tarr \& Bülthoff, 1998). To account for the present data, an instance model would need to rely primarily on recent views for the purpose of comparison with current perceptual information. Finally, previous views may be integrated or averaged to form a composite representation. Again, this composite representation would need to weight recent views more heavily than earlier views to account for the present results.

\section{CONCLUSION}

Through the extended addition of small orientation changes, participants came to perceive significantly different views of a scene as an unchanged continuation of the initial view. This sustained change blindness was caused by a dissociation between visual memory and explicit change detection: Visual memory was implicitly updated with incremental rotation to reflect recent views. Thus, the comparison between current perceptual information and scene memory operated over similar representations, even though both of these representations were often significantly different from the initial view.

\section{REFERENCES}

ChuA, K.-P., \& Chun, M. M. (2003). Implicit scene learning is viewpoint dependent. Perception \& Psychophysics, 65, 72-80.

DiWAdKaR, V. A., \& McNamaRa, T. P. (1997). Viewpoint dependence in scene recognition. Psychological Science, 8, 302-307.

FernándeZ-Duque, D., \& Thornton, I. M. (200). Change detection without awareness: Do explicit reports underestimate the representation of change in the visual system? Visual Cognition, 7, 324-344.

Hayhoe, M. M., Bensinger, D. G., \& Ballard, D. H. (1998). Task constraints in visual working memory. Vision Research, 38, 125-137.

Henderson, J. M., \& Hollingworth, A. (2003a). Eye movements and visual memory: Detecting changes to saccade targets in scenes. Perception \& Psychophysics, 65, 58-71.

HeNDERSON, J. M., \& HollingWORTH, A. (2003b). Global transsaccadic change blindness during scene perception. Psychological Science, 14, 493-497.

HOLLINGWORTH, A. (2003). Failures of retrieval and comparison constrain change detection in natural scenes. Journal of Experimental Psychology: Human Perception \& Performance, 29, 388-403.

HOLLINGWORTH, A. (2004). Constructing visual representations of natural scenes: The roles of short- and long-term visual memory. Journal of Experimental Psychology: Human Perception \& Performance, 30, 519-537.

Hollingworth, A., \& Henderson, J. M. (2002). Accurate visual memory for previously attended objects in natural scenes. Journal of Experimental Psychology: Human Perception \& Performance, $\mathbf{2 8}$, $\underline{113-136 .}$
Hollingworth, A., Williams, C. C., \& Henderson, J. M. (2001). To see and remember: Visually specific information is retained in memory from previously attended objects in natural scenes. Psychonomic Bulletin \& Review, 8, 761-768.

IRWIN, D. E. (1991). Information integration across saccadic eye movements. Cognitive Psychology, 23, 420-456.

McCloskey, M., \& Zaragoza, M. (1985). Misleading postevent information and memory for events: Arguments and evidence against memory impairment hypothesis. Journal of Experimental Psychology: General, 114, 1-16.

Mitroff, S. R., SimONS, D. J., \& Franconeri, S. L. (2002). The siren song of implicit change detection. Journal of Experimental Psychology: Human Perception \& Performance, 28, 798-815.

Mitroff, S. R., Simons, D. J., \& Levin, D. T. (in press). Nothing compares 2 views: Change blindness can result from failures to compare retained information. Perception \& Psychophysics.

O'Regan, J. K. (1992). Solving the "real" mysteries of visual perception: The world as an outside memory. Canadian Journal of Psychology, 46, 461-488.

O'REGAN, J. K., \& Nö̈, A. (2001). A sensorimotor account of vision and visual consciousness. Behavioral \& Brain Sciences, 24, $939-$ 1011.

Rensink, R. A., O'Regan, J. K., \& Clark, J. J. (1997). To see or not to see: The need for attention to perceive changes in scenes. Psychological Science, 8, 368-373.

SimONS, D. J. (1996). In sight, out of mind: When object representations fail. Psychological Science, 7, 301-305.

Simons, D. J. (2000). Current approaches to change blindness. Visual Cognition 7, 1-16.

Simons, D. J., CHABRIs, C. F., Schnur, T. T., \& Levin, D. T. (2002). Evidence for preserved representations in change blindness. $\underline{C o n-}$ sciousness \& Cognition, 11, 78-97.

Simons, D. J., Franconeri, S. L., \& Reimer, R. L. (2000). Change blindness in the absence of visual disruption. Perception, 29, 11431154 .

Simons, D. J., \& WANG, R. F. (1998). Perceiving real-world viewpoint changes. Psychological Science, 9, 315-320.

TARR, M. J., \& BülthOFF, H. H. (1998). Image-based object recognition in man, monkey, and machine. In M. J. Tarr \& H. H. Bülthoff (Eds.), Object recognition in man, monkey, and machine (pp. 1-20). Cambridge, MA: MIT Press.

Williams, P., \& Simons, D. J. (2000). Detecting changes in novel 3D objects: Effects of change magnitude, spatiotemporal continuity, and stimulus familiarity. Visual Cognition, 7, 297-322.

\section{NOTES}

1. These numbers sum to more than 25 because some participants reported more than one type of change.

2 . By updated, we mean only that the memory representation entering into comparison came to reflect recent scene information. Possible mechanisms supporting this phenomenon are discussed in the General Discussion section.

3. These results demonstrate a dissociation between explicit change detection and visual memory updating, but do they constitute an example of "implicit change detection" (Mitroff, Simons, \& Franconeri, 2002)? This depends on how one defines implicit. One cannot conclude from these data that the updating effect resulted from a different form of memory representation or a different form of comparison than those supporting explicit change detection. However, visual memory was clearly sensitive to the difference between views, and this sensitivity was implicit in the sense that it occurred despite the fact that the participants were not aware that the scene had changed.

4. Although Mitroff et al. (2002) have questioned whether "implicit" effects of change reflect implicit forms of representation and comparison, the basic fact that effects of unreported changes appear on indirect measures and, thus, that explicit report underestimates visual memory is not disputed.

(Manuscript received April 16, 2003; revision accepted for publication October 13, 2003.) 